\title{
Spectral and morpho-stratigraphic units integration on Apollo basin and Leibnitz/Von Karman craters on the Moon
}

Francesca Zambon ${ }^{1}$, Cristian Carli ${ }^{1}$, Francesca Altieri ${ }^{1}$, Jean-Philippe Combe ${ }^{2}$, Carolyn $\mathrm{H}$. van der Bogert $^{3}$, Claudia Pöler ${ }^{3}$, Harald Hiesinger ${ }^{3}$, Stephane Le Mouélic ${ }^{4}$, Nicolas Mangold ${ }^{4}$, Gwénaël Caravaca $^{4}$, and Matteo Massironi ${ }^{5}$

${ }^{1}$ INAF - IAPS, Roma, Italy (francesca.zambon@inaf.it)

${ }^{2}$ Bear Fight Institute, 22, Fiddler's Road, P.O. Box 667, Winthrop, WA, 98862, USA;

${ }^{3}$ Institut für Planetologie, Westfälische Wilhelms Universität, Wilhelm-Klemm-Str. 10, 48149 Münster, Germany

${ }^{4}$ LPG, UMR CNRS 6112, Université de Nantes, France

${ }^{5}$ Dipartimento di Geoscienze, Padova University, Padova, Italy

South Polar Aitken (SPA) is the largest and deepest basin on the Moon [1, 2], and several regions within this widespread basin have been considered as possible landing sites for present and future missions. Some of these sites are also targets of the H2020 $n^{\circ} 776276-$ PLANMAP project [3]. In this work, we focus on specific areas within SPA, Apollo basin, Lebinitz and Von Karman craters regions (see fig. 1). For our analysis, we considered the $\mathrm{M}^{3} /$ Chandrayaan-1 data [4], after the application of the last calibration, thermal removal and photometric correction $[5,6]$.

SPA is rich in high Ca-pyroxenes, except for some central crater peaks, where low Ca-pyroxenes dominate, and no extensive olivine-rich areas are observed $[1,7,8]$. Based on the spectral characteristics on the $\mathrm{M}^{3}$ spectra, we define specific spectral parameters, such as band centers, depths, spectral slopes, including selected RGB color composite combinations [5]. These indices are the starting point for retrieving the spectral units of the regions considered. The final aim of this work is to find a link between spectral, and morpho-stratigraphic units [9] to reach out highly informative geological maps of the Moon. This innovative approach holds the efforts of different research units and is one of the main goals of the PLANMAP project.

References: [1] Ohtake, M. et al., 2014, GRL. [2] Moriarty, D.P. et al., 2018, JGR. [3] https://www.planmap.eu/, [4] Pieters et al., 2009, Current Science., [5] PLANMAP D4.3- Spectral Indices and RGB maps. [6] Besse, S. et al., 2012, Icarus. [7] Dhingra, D., 2018, Geosciences. [8] Melosh, H. J., et al., 2017, Geology. [9] Ivanov, M.A., 2018, JGR.

Acknowledgments: This work is funded by the European Union's Horizon 2020 research grant agreement No 776276- PLANMAP. 

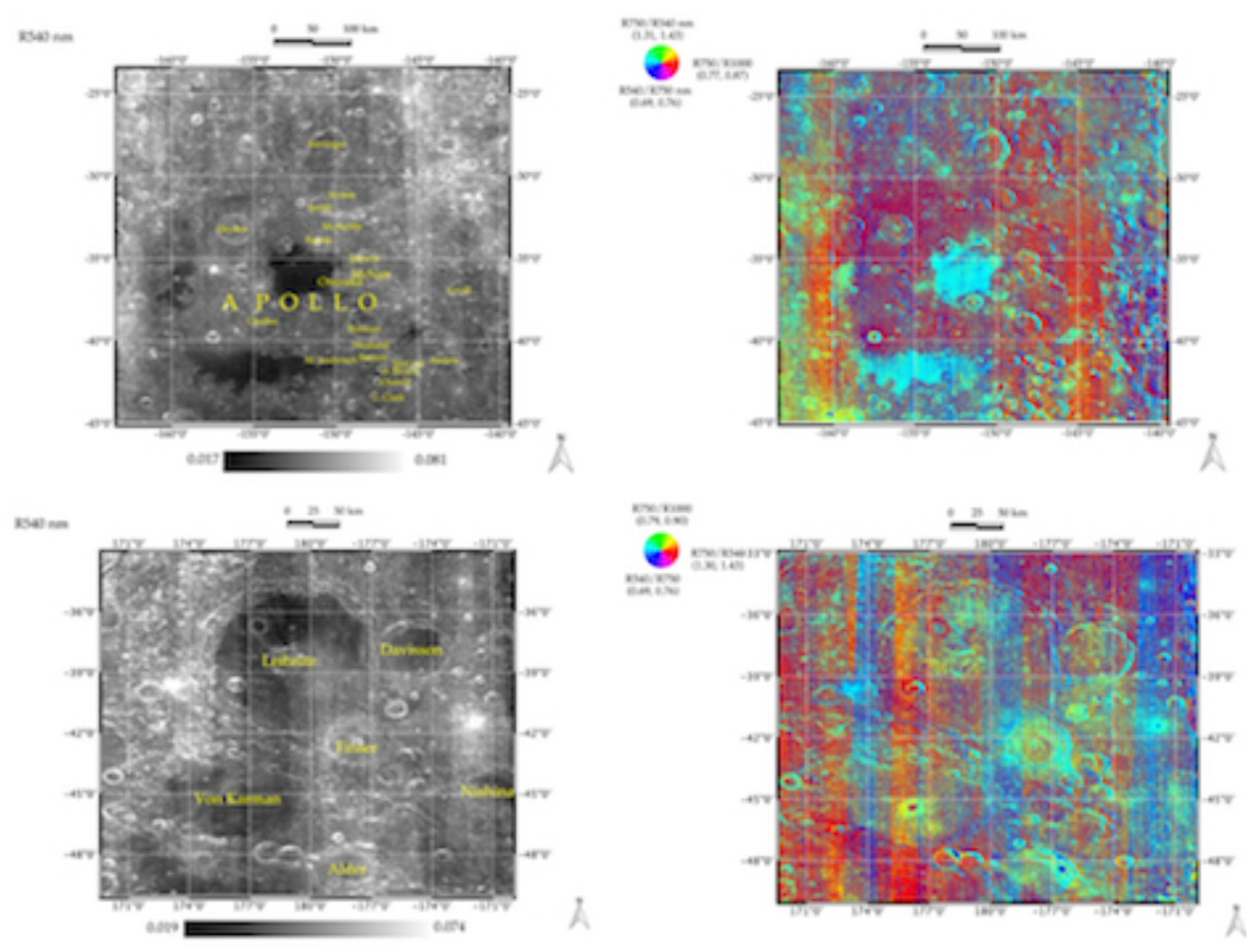

Figure 1: Apollo basin (top-left) and Leibnitz and Von Karman craters (bottom-left) reflectance map at $540 \mathrm{~nm}$. Apollo basin (top-right) and Leibnitz and Von Karman craters (bottom-right) Clementinelike mosaic derived by $\mathrm{M}^{3}$ data (bottom-right). 